quality crystals, followed by the development of estimation method of the microgravity effect on crystallization.

In the project, high viscosity of the precipitant solution had positive effects on the quality of the protein crystal grown in microgravity. We have obtained high-quality crystals of alpha-amylase diffracted to $0.89 \AA$ and of lysozyme diffracted to $0.88 \AA$ both at SPring- 8 beamline BL12B2 in the project. Both precipitant solutions contained polyethylene glycol either as a precipitant or an additive. It might be because protein and impurity depletion zones are positively formed in high viscous solutions especially in the microgravity environment.

We developed the method for estimating the diffusion coefficient (D) and kinetic coefficient $(\beta)$ by a simple experiment. The value ' $D / \beta$ ' indicates that protein and impurity depletion zone around the crystal is formed in microgravity if $D / \beta$ is low enough. Since we can predict the effects of microgravity on the protein crystal growth before performing microgravity experiment, it is possible to select samples and crystallization conditions which have high possibility to improve the crystal quality. Moreover, if we modify the crystallization condition to lower $\mathrm{D} / \beta$, the improvement of the crystal quality can be expected in microgravity experiment.

We are grateful to the Japan Synchrotron Radiation Research Institute (JASRI) for access to and user support for the synchrotron facilities of BL12B2 at SPring-8, Harima, Japan. We thank ESA and the Belgium government for Odissea mission and the usage of Granada Crystallization Facility (GCF), the Federal Space Agency and RSC Energia for the usage of the Russian Service Module, and NASA for the usage of the incubator in the US module. We would like to thank Professor Garcia-Ruiz and the members of his laboratory in CSIC-University of Granada for their helpful advices.

\section{MS06 P05}

JAXA-GCF Project --- The Past, Present and Future Masaru Sato, ${ }^{a}$ Hiroaki Tanaka, ${ }^{\mathrm{b}}$ Koji Inaka, ${ }^{\mathrm{c}}$ Shinichi Shinozaki, ${ }^{\mathrm{d}}$ Sachiko Takahashi, ${ }^{\mathrm{b}}$ Mari Yamanaka, ${ }^{\mathrm{b}}$ Erika Hirota, ${ }^{\text {b }}$ Satoshi Sano, ${ }^{a}$ Tomoyuki Kobayashi, ${ }^{a}$ Tetsuo Tanaka, a JAXA, Japan. ${ }^{b}$ Confocal Science Inc., Japan. ${ }^{\mathrm{c} M a r u w a}$ Foods and Biosciences Inc., ,Japan. ${ }^{d}$ Japan Space Forum.. E-mail: sato.masaru@jaxa.jp

Keywords: microgravity crystal growth, atomic resolution crystallography, high-resolution X-ray diffraction

Japan Aerospace Exploration Agency (JAXA) finished JAXA-GCF project in 2006. Totally six protein crystallization experimental opportunities in space were provided to crystallographers, more than 250 protein samples were launched, using Russian flight opportunities, twice a year, from 2003. In the project, the success rate of crystallization, that is mostly the improvement of the maximum resolution, has been significantly increased to about $70 \%$ of protein that was highly purified and succeeded in the optimization of the crystallization condition. Moreover, the maximum resolution was even improved if the crystal showed already an excellent resolution around $1 \AA$ in the ground-based experiment. We have obtained several know-how to grow high-quality crystals in space.
Based on them, JAXA has started JAXA-New-GCF (JAXA-NGCF) experiment in 2007. Three flight opportunities are scheduled, once in every six months. The first flight has already launched on Jan. 18, 2007 and will be landed in April. The purposes of JAXA-NGCF are to obtain atomic-resolution crystals for precise structural analysis, to cooperate with national project, and to transfer technology to private companies for commercial use.

We thank ESA and the Belgium government for Odissea mission and the usage of Granada Crystallization Facility (GCF), the Federal Space Agency and RSC Energia for the usage of the Russian Service Module, and NASA for the usage of the incubator in the US module. We are grateful to Professor Garcia-Ruiz and the members of his laboratory in CSIC-University of Granada for their helpful advices. We thank Protein 3000 Project (Riken and eight universities), NIAS, PCProt, and other users for providing protein samples.

\section{MS06 P06 \\ $\mathrm{Cu}$ co-crystallization and metal-ions cross-influence as a new optimization tools Ivana Tomčová ${ }^{\mathrm{a}, \mathrm{b}}$ and Ivana Kutá Smatanováa,b ${ }^{a}$ Institute of Physical Biology, University of South Bohemia in České Budějovice, Czech Republic. 'Institute of Systems Biology and Ecology, Academy of Sciences of the Czech Republic, Nové Hrady, Czech Republic. E-mail: tomcova@ufb.jcu.cz}

Keywords: Crystal morphology, Cupric compounds, Cross-crystallization

The effect of several metal cations $\left(\mathrm{Cu}^{2+}, \mathrm{Cd}^{2+}, \mathrm{Co}^{2+}\right.$, $\mathrm{Ba}^{2+}$ ) was tested in attempts to improve crystallization and verify a newly discovered cross-crystallization method with two selected proteins; di-heme cytochrome $c_{4}$ from anaerobic purple sulphur bacterium Thiocapsa roseopersicina and sweet-tasting protein thaumatin from the African berry Thaumatococcus daniellii. $\mathrm{Cu}^{2+}$ ions promoted the most dramatic improvement in crystal morphology, internal packing and diffraction quality. This investigation qualitatively established the influence of cupric cations on the crystal growth by using the crosscrystallization procedure.
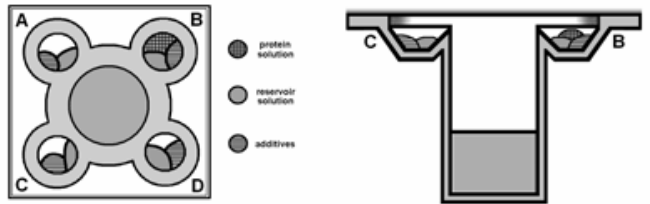

(Fig.: Schematic side and top view of Emerald BioStructures CombiClover Crystallization Plate used for sitting drop cross-crystallization experiments). It was found that influence of $\mathrm{Cu}^{2+}$ ions produced evidently different outer morphology and internal packing of thaumatin crystals (hexagonal prism). Usually their shape is presented as a tetragonal bipyramids. In the case of cytochrome, the good diffractable crystals were obtained only by using cross-crystallization method with metal-ion salts. Newly grown crystals (hexagonal prisms) of thaumatin and cytochrome displayed as the same primitive tetragonal system and diffracted up to $1.7 \AA$. Crystals were suitable for high-resolution structure analysis. (Table: Crystal morphology and internal packing influenced by metal-ion salts). 